
\title{
25 Research Soure \\ Establishment and differentiation of long-term trophoblast organoid cultures from the human placenta.
}

\section{Lucy Gardner ( $D$ Ig209@cam.ac.uk)}

Centre for Trophoblast Research, University of Cambridge

Margherita Turco ( $\nabla$ myt25@cam.ac.uk)

Centre for Trophoblast Research, University of Cambridge

Graham Burton

Centre for Trophoblast Research, University of Cambridge

Ashley Moffett

Centre for Trophoblast Research, University of Cambridge

\section{Method Article}

Keywords: human, placental, trophoblast, organoids, extraembryonic, 3-D culture, Matrigel

Posted Date: November 30th, 2018

DOI: https://doi.org/10.1038/protex.2018.121

License: (c) (i) This work is licensed under a Creative Commons Attribution 4.0 International License. Read Full License

Version of Record: A version of this preprint was published on September 9th, 2020. See the published version at https://doi.org/10.1038/s41596-020-0381-x. 


\section{Abstract}

This protocol describes the generation of long-term, genetically-stable organoid cultures of extraembryonic fetal trophoblasts from human first trimester placentas. Trophoblast organoids are consistently established within one to two weeks. They can be expanded long term, cryopreserved and resurrected. Villous cytotrophoblasts \(VCT) expressing EpCam, Ki67 and TP63 form an outer cell layer with deposition of basement membrane in contact with Matrigel. Differentiated, multinucleated syncytial cytotrophoblasts $\backslash(\mathrm{SCT})$ form in the centre of the organoid, analogous to the intervillous space. They secrete placental specific peptides and hormones such as hCG, GDF15, PSGs and PAPPA. Characteristic features of SCT are confirmed by electron microscopy $\backslash(E M)$. In addition, the organoids can be differentiated to invasive HLA-G+ extravillous trophoblasts $\backslash(E V T)$ which digest and migrate out through the Matrigel. In summary, we describe the generation of trophoblast organoids from human extraembryonic tissues that grow as complex 3D structures with fusion of VCT to hCG-secreting SCT, anatomically and functionally closely resembling the villous placenta in vivo.

\section{Introduction}

A long-term in vitro model representative of normal bone fide human trophoblasts has long been sought by researchers studying human placentation. This organoid model generates villous cytotrophoblasts, syncytiotrophoblasts and extravillous cytotrophoblasts, the three main trophoblast sub-populations comprising the first trimester placenta. The model will enable investigation of proteins and hormones produced by SCT which play a role in the pathogenesis of common, systemic, pregnancy disorders including gestational diabetes, hepatic cholestasis, pre-eclampsia and hyperemesis gravidarum. Characterisation of placental products will now be possible. The major, unexplained disorders of pregnancy, pre-eclampsia, stillbirth and fetal growth restriction are thought also to have their origins in aberrant placental development during the first ${ }^{1}$. In this regard, the organoids will be useful for studying factors which influence EVT development and function. Factors which are encountered in vivo in the decidual microenvironment, such as the products of glandular histotrophic nutrition and interaction with the distinctive resident uterine natural killer $\backslash(N K)$ cell population are of interest for investigation using this model ${ }^{2,3}$. Trophoblast organoids can therefore be used to study both human placental development and interactions with the local and systemic maternal environment. They will be a readily accessible substitute for freshly isolated primary cells.

\section{Reagents}

-HAMS/F12 medium \(Biosera \#SM-H0096) • Penicillin/ streptomycin solution \(Sigma, P0781) • trypsin$250 \backslash($ Pan Biotech P10-025100P) • EDTA \(Sigma \#E9884) • Collagenase V \(Sigma \#C9263) • Fetal calf serum \(FCS) \(Biosera \#FB-1001 or similar) • Thawed Matrigel matrix \(Corning \#356231) on ice. • Advanced DMEM/F12 medium \(Life Technologies, 12634010) • N2 supplement \(Life Technologies, 17502048) • B27 supplement minus vitamin A \(Life Technologies, 12587010) • Primocin \(Invivogen, ant-pm-1) • N-Acetyl-L-cysteine $\backslash($ Sigma, A9165-5G) • L-glutamine $\backslash($ Sigma, 25030-024) • Recombinant 
human EGF \(Peprotech, AF-100-15) • CHIR99021 \(Tocris, 4423) • Recombinant human Rspondin-1 \ (Peprotech, 120-38) • Recombinant human FGF-2 \(Peprotech, 100-18C) • Recombinant human HGF $\backslash$ (Peprotech, 100-39) • A83-01 \(Tocris Biotechne \#2939) • Prostaglandin E2 \(Sigma, P0409) • Y-27632 \ (Millipore, 688000) • 2-mercaptoethanol \(Gibco 31350) • BSA \(Sigma A8412) • ITS-X supplement \ (Gibco 51500-056) • NRG1 \(Cell signalling 5218SC) • KO serum replacement $\backslash($ ThermoFisher \#10828010).

\section{Equipment}

- Samples of placental tissue from 6-9 week gestation obtained with written informed patient consent. • Category 2 microbiological safety cabinet. \(Envair, Eco safe comfort plus SCS-EVO-2.4). N Nitrile, powder free examination gloves $\backslash($ Kimtech, 90627). • Cell culture humidified C02 incubator 370c, 5\%CO2 \ (Thermo Scientific, Heraeus BB15, 1284-6716) - Heater shaker set at 370C $\backslash($ Barnstead International, Max Q mini 4000, SHKA4450-1CE) • Digital inverted microscope \(Life Technologies, Evos XL Core, AMEX1000) - Sterile dissection forceps $\backslash($ Scientific Laboratory Supplies, SR04010) $\cdot 140 \mathrm{~mm}$ petri dishes $\backslash($ Sterilin, SC269) • Disposable, sterile No.22 scalpels $\backslash($ Swann Morton, 0508) • Magnetic stirrer $\backslash($ Stuart, US152) - Sterile magnetic stirrer bars $\backslash($ Scientific Laboratory Supplies, STI5016) • Sterile $250 \mathrm{ml}$ glass Duran bottles $\cdot 60 \mathrm{ml}$ sterile specimen containers $\backslash($ Elkay, 500-3000-065) $\cdot$ sterile muslin gauze $\backslash($ Winware food grade, E948) • Disposable sterile 3ml plastic Liquipette \(Elkay, 127-P503-STR) • Microcentrifuge tubes, $1.5 \mathrm{ml}$, sterile $\backslash($ Starlab, S1615-5500) • Low bind Eppendorf tubes, $1.5 \mathrm{ml}$, sterile $\backslash$ (Eppendorf, Protein

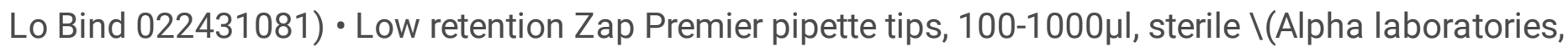

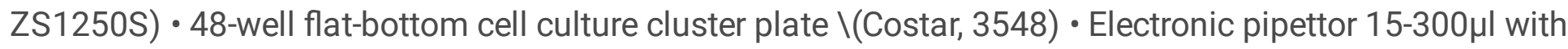
mix function \(Eppendorf, Xplorer Plus 613-2231) • CryoTube vials 1.8ml $\backslash($ Thermo Scientific Nunc, 377267) • Benchtop centrifuge $\backslash($ Eppendorf, 5702, rotor A-4-38) • 30ml Universal tubes $\backslash($ Sterilin, 128A/P) - Small bore $200 \mu$ l pipette tips, sterile $\backslash($ Rainin, BioClean GP-200F) • Mr. FrostyTM freezing container $\backslash$ (Thermo Fisher Scientific, 5100-0001) • Automatic Pipettor \(Integra PIPETGIRL, 155021) • Sterile 10ml disposable pipettes $\backslash($ Greiner 607-180) • 1-channel Manual pipettes $\backslash($ Sartorius, Tacta 2-20 $\mathrm{ll}$ LH-729030,

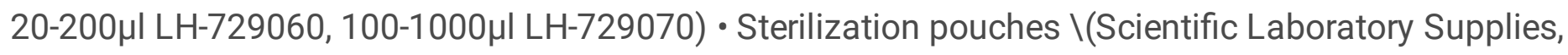
AUT1556) • ibidi $\mu$-dishes $\backslash($ Thermo Scientific \#81156)

\section{Procedure}

**Initiation of culture**. Tissues should be regarded as potentially infected with hazard group 3 blood borne pathogens and should be handled with appropriate personal protective equipment. 1 Transfer placental samples into basic wash medium plus penicillin/streptomycin at room temperature and process within one hour. 2 Pre-warm 0.2\% trypsin-250 \(Pan Biotech P10-025100P)/0.02\% EDTA \(Sigma \#E9884)/PBS to $370 \mathrm{c}$ in a water bath. 3 Wash the placental tissue in basic culture medium with gentle stirring for $>10$ minutes to remove contaminating blood and other maternal uterine cells. 4 Tip into a petri dish. Transfer washed placental pieces with forceps to a clean dish. 5 Scrape villi from the chorionic membrane with a scalpel. Discard membrane. 6 Transfer villi to $50-70 \mathrm{ml}$ pre-warmed $0.2 \%$ trypsin- 
250/0.02\% EDTA in a bottle with a magnetic stirrer. Seal the lid and place in a heated shaker at $37 \mathrm{C}$ with gentle shaking to digest for 3-5 min. 7 Filter the disaggregated cell suspension through sterile muslin gauze and wash through immediately with Hams F12/20\% FBS to arrest trypsin digestion. \(Retain the gauze with partially digested remnants). 8 Centrifuge the filtrate and re-suspend the cell pellet in Hams F12 medium. 9 Retrieve undigested tissue from the gauze and digest further with 10-15 ml collagenase $V$ solution $1.0 \mathrm{mg} / \mathrm{ml}$ in Hams $\mathrm{F} 12 / 10 \%$ FBS with gentle shaking at $37^{\circ} \mathrm{C}, 5 \mathrm{~min}$. 10 Filter the resultant cell suspension and centrifuge to pellet cells as before. 11 Pool the cells from both digests and wash in Advanced DMEM/F12 medium. 12 Resuspend pellet in 1ml Advanced DMEM/F12 medium, transfer to $1.5 \mathrm{ml}$ Eppendorf tube and pellet again by centrifugation. 13 Remove the supernatant and estimate the volume of the pellet. Flick the tube to loosen the pellet and place on ice for 2-3 minutes. Add $10 x$ volume:volume of ice-cold Matrigel and pipette gently to mix. Place tube immediately back onto ice. 14 Plate 20-25 $\mu \mathrm{l}$ drops of Matrigel/cell suspension into the centre of wells of Corning 48-well tissue culture

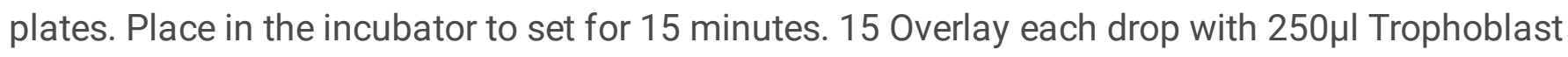
Organoid Medium \(TOM). 16 Maintain cultures in $5 \% \mathrm{CO}_{2}$ in a humidified incubator at $37^{\circ} \mathrm{C}$. Replace medium every 2-3 days. 17 Examine cultures for the appearance of small organoid clusters. These are usually visible by $\sim 7$ days and continue to grow and self-organise. They can be passaged as below when at least $50 \%$ reach a diameter of 200-300 $\mu \mathrm{m} \backslash$ (usually between another 7-10 days). **Passaging ${ }^{\star \star} 1$ Without removing the culture medium, using a $1 \mathrm{ml}$ pipette tip scrape backwards and forwards across the growth surface of each well to detach the Matrigel drop into the medium. Transfer the contents of the wells into $1.5 \mathrm{ml}$ Eppendorf tubes at a ratio of 4 wells per tube. 2 Centrifuge at $600 \mathrm{~g}, 6$ minutes to pellet. Remove the supernatant and add approximately $150 \mu$ I Advanced DMEM F12 to each tube. Pipette up and down 400 times through a small bore pipette tip to break up the organoids and the Matrigel. Do not use excessive force. An electronic pipettor with a 'mix' function and its uptake volume set to $150 \mu \mathrm{l}$ is recommended for this step. 3 Add $1 \mathrm{ml}$ Advanced DMEM F12 and centrifuge at $600 \mathrm{~g}, 6 \mathrm{~min}$. Remove supernatant. Add 150ul Advanced DMEM F12 and pipette up and down manually a further 80 times using moderate force. 4 Add $1 \mathrm{ml}$ Advanced DMEM F12 and centrifuge at $600 \mathrm{~g}, 6 \mathrm{~min}$. Remove supernatant. The pellet at this stage should contain very little Matrigel. If Matrigel is still present, estimate the volume. Flick the tubes to re-suspend the pellets. Place tubes on ice. 5 Working one tube at a time,

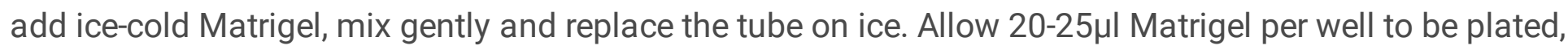
remembering to subtract the estimated volume of any residual Matrigel which remained in the pellet. Pool organoids together and mix gently to distribute them evenly throughout the Matrigel. 6 Dispense $25 \mu \mathrm{l}$ drops carefully onto the centre of the growth area of wells of a 48-well culture plate, taking care not to create bubbles. 7 Place culture plate in the incubator for $15 \mathrm{~min}$ to allow Matrigel to set. $8 \mathrm{Add} 250 \mu \mathrm{l}$ trophoblast Organoid Medium \(TOM) to each well. **Freezing ${ }^{\star \star} 1$ Place $20 \%$ DMSO freeze medium on ice to cool. Label cryovials. 2 Without removing the culture medium, using a $1 \mathrm{ml}$ pipette tip scrape backwards and forwards across the growth surface of each well to detach the Matrigel drop into the medium. Transfer the contents of the wells into $1.5 \mathrm{ml}$ Eppendorf tubes at a ratio of 3-4 wells per tube. 3 Centrifuge $600 \mathrm{~g}, 6$ minutes to pellet organoids. 4 Remove supernatant and add 200 $\mu$ Advanced DMEM F12 to each tube. Manually pipette gently up and down 80 times with moderate force to partially disrupt organoids. Add 1ml Advanced DMEM F12 and centrifuge again. 5 Remove supernatant and flick pellets 
to re-suspend. Add 500ul TOM/40\% FBS per tube and cool tubes on ice. 6 Slowly add 500 ul cold $20 \%$ DMSO freeze medium per tube with gentle mixing. 7 Transfer contents to labelled cryovials on ice. Transfer immediately to 'Mr. Frosty' or similar cell freezing vessel at minus 800c overnight, then to liquid nitrogen for long-term storage. ${ }^{*}$ Generation of EVT from trophoblast organoids ${ }^{\star \star}$ Differentiation was accomplished using a modification of the protocol used by Okae et al. ${ }^{4} .1$ Passage organoids and replate in several Matrigel drops into ibidi $\mu$-dishes. 2 Culture for 3-4 days in TOM. 3 Change medium to EVT medium \#1. Culture for several days, replenishing medium every 2-3 days. 4 When adherent outgrowths begin to appear \(typically 7-10 days) change medium to EVT medium \#2. 5 Continue culturing in EVT medium \#2 for a further 7-10 days or until sufficient numbers of adherent EVT have been generated. 6 Expression of HLA-G protein by immunofluorescence or flow cytometry can be used to verify differentiation to EVT.

\section{Timing}

Initiation of culture - $1 \frac{1}{2}$ hours from receipt of tissue into the lab. Passaging $-1 \frac{1}{2}$ hours. Freezing - 1 hour.

\section{Troubleshooting}

1 Despite extensive washing of the placental tissue samples, occasional contamination of placental organoid cultures by cystic glandular organoids occurred. These were confirmed to be of maternal origin by HLA typing and microsatellite analysis. These cultures should be discarded. This indicates that contamination originating from close intermingling of maternal and fetal cells in utero can lead to the generation of both maternal glandular and fetal trophoblast organoid cultures from the same tissue. 2 Organoids freed from Matrigel are prone to adhere to the surface of plastic tubes resulting in reduction of yield. When the numbers of organoids are low, loss can be minimised by the use of low protein-binding tubes and low-retention pipette tips. 3 It is important during plating to keep the Matrigel/cell suspension on ice as it quickly sets at room temperature. 4 Organoids are best propagated by passaging before they become too large. They will however survive and continue to grow in size if observation of longer-term self-organization is desired.

\section{Anticipated Results}

We have been successful in generating pure placental organoids from $90 \%$ of 6-9 week gestation placental samples processed within two hours of receipt.

\section{References}

1. Smith, G. C. First-trimester determination of complications of late pregnancy. JAMA 303, 561-562 \} (2010). 2. Burton, G. J., Jauniaux, E. \& Charnock-Jones, D. S. The influence of the intrauterine environment on human placental development. Int J Dev Biol 54, 303-312 \(2010). 3. Moffett, A. \& Colucci, F. Coevolution of NK receptors and HLA ligands in humans is driven by reproduction. Immunol. Rev. 267, 283- 
$297 \backslash(2015) .4$. Okae, H. et al. Derivation of Human Trophoblast Stem Cells. Cell Stem Cell 22, 50-63 e6 \ (2018).

\section{Acknowledgements}

The authors are grateful to patients for donating tissue for research. We thank D. Moore and staff at the Addenbrookes Hospital, Cambridge. This work was funded by Centre for Trophoblast Research, University of Cambridge and Medical Research Council \(MR/L020041/1). A.Moffett holds a Wellcome Trust joint investigator award $\backslash(200841 / Z / 16 / Z)$. M.Y.Turco received funding from E.U. 7th Framework Programme for research, technological development and demonstration \(PIEF-GA-2013-629785) and now holds a Royal Society Dorothy Hodgkin Fellowship. 